\title{
BOUNDARY LAYERS IN EXTRAGALACTIC JETS
}

\author{
DAVID S. DE YOUNG \\ Kitt Peak National Observatory \\ Tucson, AZ USA
}

\section{Introduction}

The radio morphology of extragalactic radio sources, in particular the FR I objects, has long been recognized to be suggestive of an outflow which is undergoing a strong interaction with the surrounding medium. Two essential elements must be noted in considering this interaction. First, it is inevitable. Second, the interaction will always provide mass and energy transfer from the jet to the ambient medium, and thus it is a loss term in the overall energy balance of the radio source. As will be seen, these losses can be comparable to the overall radio luminosity and hence are a nontrivial constraint on the energy requirements placed on the central engine.

The onset of boundary layer development is driven by the instability of the shear layer between the jet and the surrounding medium. An important experimental result is that such layers are characterized by the growth and persistence of large scale structures within the layer. These structures grow roughly linearly with time in the early stages, and they are the objects which mediate the mass entrainment and energy transfer between the jet and its surroundings. Empirically, the entrainment process appears to be one of "gulping" followed by interior mixing. The experimental data have not given rise to a comprehensive theoretical understanding of this highly nonlinear phenomenon.

\section{A New Model for Boundary Layer Development}

Consideration of the development of the layer begins in the early nonlinear stage of growth, where small and well separated vortex structures have already formed. The basic questions are the location of the entrainment, the rate of entrainment, and the growth rate of the large structures. Exami- 
nation of the empirical data on such boundary layers reveals the following self consistent solution.

On small scales where the vortex size is much less than the jet radius a two dimensional approximation will be appropriate. Between the growing vortices the flow converges from either side to a contact discontinuity between the jet and the surroundings. This flow field places a stagnation point at a location on the contact surface midway between adjacent vortices. Mass flows into the vortices along the contact surface. The initial distance between the small vortices is $2 a$, and the initial vortex radius is set at some $R_{o}$. The ansatz is now to set the azimuthal velocity beyond $R_{o}$ equal to that created by a point vortex. i.e., far from the vortex the fluid has a velocity field with magnitude decreasing as $1 / r$. This velocity field then determines the problem for divergence free flow. Material flows from the stagnation point into the vortex with the mass flow rates given by the flow field. The vortex growth rate is then given by conservation of mass. Thus per unit thickness $\Delta z$ transverse to the flow the mass inflow is

$$
d M / d t=\rho_{o} \Delta z \int_{R}^{a} v_{\theta}(r) d r .
$$

The corresponding vortex growth rate is given by

$$
d R / d t=(1 / 2 \pi) v_{\theta}\left(R_{o}\right) \ln (a / R) .
$$

The equation for the vortex radius as a function of time has no closed analytic solution but can be easily integrated numerically. It is obvious that the growth rate will slow as the vortex radius approaches the initial separation $2 a$. This approach has been checked by calculating the evolution of a shear layer using kinematic arrays of point vortices. A simulation employing 12000 point vortices shows that stagnation points do arise between the large scale structures as they grow.

\section{Results}

With these results one can estimate the total mass entrainment rate into a jet by "wrapping" the two dimensional structure onto a jet surface. A minimum entrainment rate results if the process is halted when the vortices grow to fill the separation between them. Using jet velocities of 10,000 $\mathrm{km} / \mathrm{s}$, a jet radius of $100 \mathrm{pc}$, and an ambient number density of 0.01 gives entrainment rates of a few solar masses per year. The kinetic energy lost to the ambient medium by this process is in this case $3 \times 10^{43} \mathrm{erg} / \mathrm{s}$, which is comparable to FR I radio luminosities. Hence the boundary layer interaction can place overall energy demands on the nuclear engine which are comparable to the radio emission and which will decelerate the source and heat its surroundings. 